\title{
Las NIC - NIIF dentro del proceso contable
}

DOI: https://doi.org/10.33262/ap.v3i3.1.77

\section{c) (1)}

\section{IAS - IFRS within the accounting process}

\author{
Norma del Rocío Toledo Castillo. ${ }^{1}$, Isabel Romané Peñafiel Moncayo. ${ }^{2} \&$ Yolanda \\ Tatiana Carrasco Ruano. ${ }^{3}$
}

\begin{abstract}
The accounting process within the entities has changed over time and has been modernized, thus in Ecuador initially the process was regulated by the NEC (Ecuadorian Accounting Standards), to later be replaced by the IAS (International Accounting Standards) and IFRS (International Financial Reporting Standards). Objectives: this work aims to identify the current accounting regulations within the accounting process. Methodology: it applies the quantitative method, bibliographic design, documentary type, cross-sectional, analytical explanatory with the objectives of the study by the causeeffect method, without the intervention of the researcher. Contribution: the theory will be related to the accounting practice, trying to establish a documentary basis for the application of the accounting process in the institutions of Ecuador.
\end{abstract}

Keywords: IAS, IFRS, processes, accounting, standards.

\section{Resumen}

El proceso contable dentro de las entidades ha cambiado a través del tiempo y se ha modernizado, así en el Ecuador inicialmente el proceso era normado por las NEC (Normas Ecuatorianas de Contabilidad), para posteriormente ser reemplazadas por las NIC (Normas Internacionales de Contabilidad) y las NIIF (Normas Internacionales de Información Financiera). Objetivos: el presente trabajo pretende identificar la normativa

\footnotetext{
${ }^{1}$ Escuela Superior Politécnica de Chimborazo, Facultad Administración de Empresas. Riobamba, Ecuador. norma.toledo@espoch.edu.ec, https://orcid.org/0000-0003-1116-760X

${ }^{2}$ Escuela Superior Politécnica de Chimborazo, Facultad Administración de Empresas. Riobamba, Ecuador. romane.peñafiel@espoch.edu.ec, https://orcid.org/0000-0002-2629-8576

${ }_{3}^{3}$ Ciencia Digital Editorial, Ambato, Ecuador, tatianacarrasco@cienciadigital.org, https://orcid.org/00000002-5294-8202
} 
contable vigente dentro del proceso contable. Metodología: aplica el método cuantitativo, diseño bibliográfico, tipo documental, de corte transversal, analítico explicativo con los objetivos del estudio por el método de causa efecto, sin intervención del investigador. Aporte: se relacionará la teoría con la practica contable, pretendiendo establecer una base documental para la aplicación del proceso contable en las instituciones del Ecuador.

Palabras claves: NIC, NIIF, procesos, contable, normas.

\section{Introducción}

El conjunto de normas contables que están aceptadas y son usadas en la gran mayoría de países son emitidas por "The International Accounting Standard Committee" (IASC), cuya sede se encuentra en Inglaterra y fue creado en el año de 1973. Este organismo es el responsable de la emisión y actualización continua de la normativa contable.

Todo el proceso contable involucra al contador, evidenciado los hechos económicos conforme a su perfil lo exige, para asegurar que la información se presente de manera confiable, fiable, oportuna y siempre reflejando la realidad de la actividad económica (Albero et al, 2021).

A nivel de Latinoamérica las NIC (Normas Internacionales de Contabilidad) - NIIF (Normas Internacionales de Información Financiera), se aplicaron desde el año 2009 (A. Fierro y F. Fierro, 2016).

Así al principio el IASB emitió veintiséis normas, que se actualizan y derogan para adaptarse a los nuevos requerimientos de información financiera. Al principio estas normas se denominaron NIC, y las nuevas normas emitidas se denominan NIIF, muchas NIC fueron derogadas y reemplazadas por las NIIF. Cuando una nueva norma se emite, su aplicación no es inmediata, generalmente demora dos años o más para entrar vigencia, y los procesos contables contemplen los nuevos requerimientos de información.

Al no estar claro como intervienen las normas en el proceso contable, debido a la gran cantidad de información que existe para aplicar, el presente trabajo pretende una guía rápida para poder aplicar las NIC - NIIFS, identificando con claridad las normas básicas en cualquier tipo de actividad económica.

En el Ecuador a partir del año 2008, entraron paulatinamente las empresas grandes, continuando las medianas, hasta que finalmente en el año 2012 todos los grupos económicos implementaron las NIC - NIIFS. A pesar del tiempo prudencial que existió para que las empresas ecuatorianas adopten la normativa, han transcurrido varios años y los inconvenientes al momento de su aplicación no han cesado.

A pesar de los obvios inconvenientes al momento de la aplicación de la norma NIIF, el Ecuador no puede quedar fuera de su aplicación, al formar parte de la gran economía 
mundial, y se acogió a la misma como lo hicieron la mayoría de países, como se puede evidenciar en la figura 1.

Figura 1

Aplicación de NIIFS en el mundo

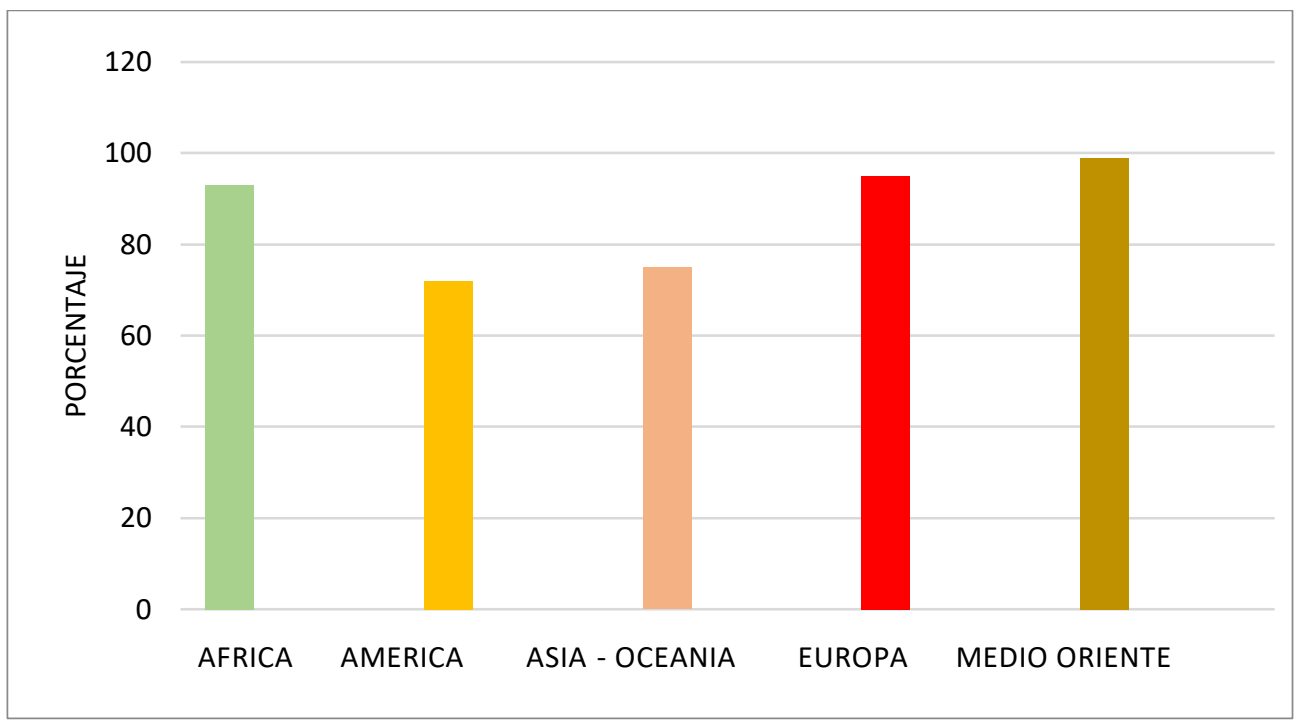

Nota: progreso hacia los estándares mundiales, 144 jurisdicciones requieren el uso de norma NIIFS para todas o la mayoría de empresas que cotizan en bolsa.

Fuente: Normas Internacionales de Información Financiera (NIIF, 2021).

\section{Metodologia}

El estudio y comprensión de los problemas humanos exige una amplia pluralidad metodológica, seleccionar uno u otro método a utilizar, dependerá del problema concreto a evaluar (Palella y Martins, p. 42).

La justificación del problema, indica la razón por la cual se quiere realizar dicha investigación, y el aporte que se pretende lograr en los distintos ámbitos, sean estos: sociales, políticos, económicos, humanos, etc. (Palella y Martins, p. 60).

Al definir los objetivos se determina qué tipo de investigación se realizará y deberá establecerse: el diseño, tipo, nivel y modalidad de estudio (Palella y Martins, p. 84).

La presente investigación aplica el método cuantitativo, diseño bibliográfico, tipo documental, de corte transversal, analítico explicativo con los objetivos del estudio por el método de causa efecto, sin intervención del investigador. La justificación del problema realiza un enfoque teórico, al analizar la normativa actual y su participación dentro del proceso contable.

\section{Marco Teórico}

Las NIIF se han convertido en el lenguaje global de facto de la información financiera, que se utiliza ampliamente en las economías desarrolladas, emergentes y en vías de desarrollo (NIIF, 2021). 
Los procesos contables dentro de una organización, expresan las políticas, procedimientos, controles; disminuyendo los desaciertos y presentando razonabilidad a los estados financieros (Valdez et al., 2019, pág. 2).

Los procedimientos económicos de una entidad para la presentación de estados financieros, forman parte del proceso contable de la institución, son actividades que la ser identificadas en un proceso rutinario establecen las políticas para los procesos (Barreto y Teodomiro, 2018).

El IASB con sede en Inglaterra genero una serie de normas NIIF, mismas que se componen por NIC, NIIF, y sus interpretaciones a las NIC y NIIF. Cada año estas normas van variando, se incrementan nuevas NIIF que reemplazan a una o varias NIC o NIIF, es por ello que la numeración de las normas no es consecutiva, así en el año 2021 tenemos la siguiente composición de la normativa.

Tabla 1

Composición de la normativa NIIF 2021

Marco Conceptual

NIIF
- NIIF 1 Adopción por primera vez de las Normas Internacionales de Información Financiera

- NIIF 2 Pagos basados en acciones

- NIIF 3 Combinación de negocios

- NIIF 5 Activos no corrientes mantenidos para la venta y operaciones discontinuadas

- NIIF 6 Exploración y evaluación de recursos minerales

- NIIF 7 Instrumentos financieros: divulgaciones

- NIIF 8 Segmentos operativos

- NIIF 9 Instrumentos financieros

- NIIF 10 Estado financiero consolidado

- NIIF 11 Arreglos conjuntos

- NIIF 12 Información a revelar sobre participaciones en otras entidades

- NIIF 13 Medición del valor razonable

- NIIF 14 Cuentas regulatorias diferidas

- NIIF 15 Ingresos por contratos con clientes

- NIIF 16 Arrendamientos

- NIIF 17 Contratos de seguros

\section{NIC}

- NIC 1 Presentación de estados financieros

- NIC 2 Inventarios

- $\quad$ NIC 7 Estado de flujos de efectivo

- NIC 8 Políticas contables, cambios en las estimaciones contables y errores

- $\quad$ NIC 10 Eventos posteriores al período sobre el que se informa

- NIC 12 Impuestos sobre la renta

- NIC 16 Propiedad, Planta y Equipo

- NIC 19 Beneficios para empleados

- NIC 20 Contabilización de las subvenciones del gobierno y divulgación de la asistencia del gobierno

- NIC 21 Los efectos de los cambios en los tipos de cambio de divisas

- $\quad$ NIC 23 Costos de préstamos

- $\quad$ NIC 24 Divulgaciones de partes relacionadas

- NIC 26 Contabilidad y presentación de informes por planes de beneficios por jubilación

- NIC 27 Estados financieros separados

- NIC 28 Inversiones en asociadas y negocios conjuntos 
Tabla 1

Composición de la normativa NIIF 2021 (continuación)

\section{Interpretaciones CINIIF}

- CINIIF 1 Cambios en pasivos existentes por desmantelamiento, restauración y similares

- CINIIF 2 Participaciones de los socios en entidades cooperativas e instrumentos similares

- CINIIF 5 Derechos de Intereses derivados de Fondos de Desmantelamiento, Restauración y Rehabilitación Ambiental

- CINIIF 6 Pasivos que surgen de la participación en un mercado específico: equipos eléctricos y electrónicos de desecho

- CINIIF 7 Aplicación del enfoque de reexpresión según la NIC 29 Información financiera en economías hiperinflacionarias

- CINIIF 10 Informes financieros intermedios y deterioro

- CINIIF 12 Acuerdos de concesión de servicios

- CINIIF 14 NIC 19 - El límite de un activo de beneficio definido, requisitos mínimos de financiación y su interacción

- CINIIF 16 Coberturas de una inversión neta en una operación en el extranjero

- CINIIF 17 Distribuciones de activos distintos al efectivo a los propietarios

- CINIIF 19 Extinción de pasivos financieros con instrumentos de patrimonio

- CINIIF 20 Costos de desmonte en la fase de producción de una mina a cielo abierto

- CINIIF 21 Tasas

- CINIIF 22 Transacciones en moneda extranjera y contraprestación anticipada

- CINIIF 23 Incertidumbre sobre los tratamientos del impuesto sobre la renta

\section{Interpretaciones SIC}

- NIC 29 Información financiera en economías hiperinflacionarias

- NIC 32 Instrumentos financieros: Presentación

- NIC 33 Ganancias por acción

- $\quad$ NIC 34 Información financiera intermedia

- $\quad$ NIC 36 Deterioro de activos

- NIC 37 Provisiones Pasivos contingentes y activos contingentes

- $\quad$ NIC 38 Activos intangibles

- NIC 39 Instrumentos financieros: reconocimiento y medición

- $\quad$ NIC 40 Propiedad de inversión

- NIC 41 Agricultura

- $\quad$ SIC-7 Introducción del euro

- $\quad$ SIC-10 Asistencia del gobierno: sin relación específica con las actividades operativas

- $\quad$ SIC-25 Impuestos sobre la renta: cambios en la situación fiscal de una entidad o sus accionistas

- $\quad$ SIC-29 Acuerdos de concesión de servicios: divulgaciones

- $\quad$ SIC 32 Activos intangibles: costos del sitio web

\section{Fuente: NIIF (2021)}

La información financiera parte de una Hipótesis Fundamental, antes de la aplicación de cualquier norma, es necesario que los estados financieros se preparen en base a las siguientes hipótesis:

- Devengo: los efectos de las transacciones se reconocen cuando ocurren, independientemente de cuando se realice su cobro o su pago,

- Empresa en funcionamiento: esta hipótesis supone que la empresa está en funcionamiento y continuara sus actividades operativas dentro de un futuro previsible.

Toda la información emitida bajo la normativa NIIF, está enfocada a emitir estados financieros para la economía mundial, y la ayuda en la toma de decisiones en las finanzas corporativas y la discusión sobre la colocación de inversiones, que intentan colocar 
recursos financieros escasos tratando de obtener un equilibrio entre rentabilidad, riesgo y liquidez (Macedonio y García, 2020).

Pero toda esta toma de decisiones se verá afectada por el método de valoración que el profesional contable selecciono para la preparación de los estados financieros. Las empresas generalmente utilizan el coste histórico; este se combina con otras bases de valoración de cuentas específicas. Además, algunas empresas usan el coste corriente como respuesta a la incapacidad del modelo contable del costeo histórico (Amat y Puig, (2018).

En la tabla 2, se muestra la clasificación de los principales grupos de los estados financieros y sus métodos de valoración.

Tabla 2

Métodos de valoración de los elementos de los estados financieros

\begin{tabular}{ll}
\hline \multicolumn{1}{c}{ Método } & \multicolumn{1}{c}{ Activos } \\
\hline $\begin{array}{l}\text { Coste Histórico } \\
\text { (Valor de adquisición) }\end{array}$ & $\begin{array}{l}\text { Se registran por el importe de } \\
\text { efectivo y otras partidas } \\
\text { pagadas, o por el valor } \\
\end{array}$ \\
& razonable, de la contrapartida \\
& entregada a cambio en el \\
& momento de la adquisición.
\end{tabular}

Coste Corriente

(Valor que constaría en la actualidad)

Valor Realizable

(Valor de liquidación actual)
Se registran por el importe de tesorería y otros medios líquidos, que deberían pagarse si se adquiriese en la actualidad el mismo activo u otro equivalente.

Se registra el valor presente, descontando las entradas netas de efectivo que se espera que genere la partida en el curso normal de la operación.
Pasivos

Se registro por el valor del producto recibido a cambio de incurrir en la deuda o, en algunas circunstancias (por ejemplo, en el caso de los impuestos), por las cantidades de tesorería y otros medios líquidos que se espera pagar para satisfacer la correspondiente deuda en el curso normal de la operación.

Se registran por sus valores de liquidación, es decir por los importes sin descontar de tesorería u otros medios líquidos, que se espera que puedan satisfacer las deudas en el curso normal de la operación.

Se registra por el valor presente, descontando las salidas netas de tesorería que se espera necesitar para pagar las deudas en el curso normal de la operación.

Fuente: Vazquez et al. (2013)

La información de los estados financieros además debe cumplir con ciertas características, que son atributos esenciales que se esperan encontrar en la emisión de la información financiera, y son:

- comprensibilidad,

- relevancia (materialidad),

- fiabilidad (representación fiel, esencia sobre la forma, integridad, neutralidad, prudencia),

- comparabilidad. 
Una de las normas generales fundamentales es la NIIF 13, medición del valor razonable. En esta norma se hace alusión a cuatro métodos para la valoración contable de transacciones

- Costo histórico, de acuerdo a como ocurrió en el momento de realización de la transacción.

- Costo corriente, registro contable según el valor actual o en el presente.

- Valor realizable, equivale a lo que podría obtenerse en el mercado en el momento de su valuación.

- Valor presente, monto actual descontado el efectivo percibido desde el inicio de su existencia hasta su liquidación (NIIF, 2021).

\section{Discusión}

La normativa NIIF aporta una base sólida para establecer un proceso contable, sin embargo, la misma permite diversos métodos de valoración de las cuentas, que combinadas con las restricciones con la aplicación tributaria, genera un sin número de posibles resultados dependiendo del método de valoración y registro que selecciono el profesional contable.

Además, se deberán tomar en cuenta las diferencias temporarias o permanentes que se generarán por la aplicación propia de la normativa.

Cualquiera de las políticas seleccionadas por el profesional contable se consideraría adecuada y dependerá mucho de la visión de la gerencia en cuanto al cumplimiento de sus objetivos.

Como se estableció en el marco teórico la normativa expide una norma para cada cuenta de los estados financieros. Sin embargo, existe una categoría de normas que de ser colocadas en una pirámide por su importancia desde la cúspide se mostrarían como se muestra en la figura.

\section{Figura 2}

Categorización de las NIIF

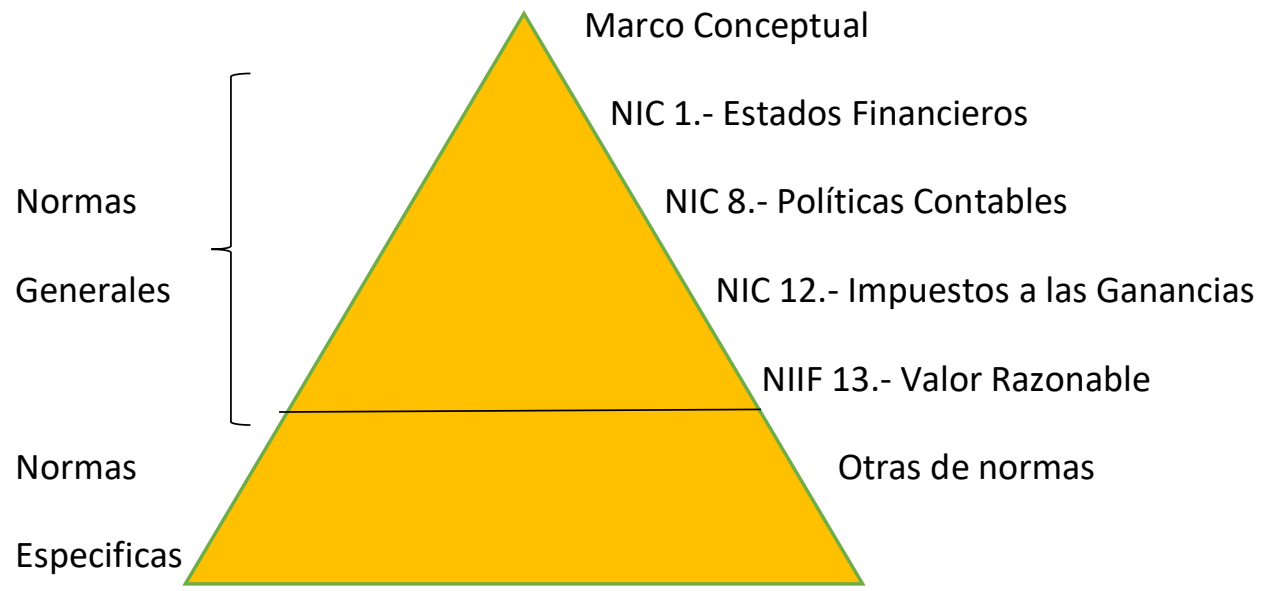

Nota: Las normas generales se aplican en todos los tipos de actividades económicas y supeditan las normas específicas

Fuente: Elaboración propia. 
Tomando en cuenta la categorización de la normativa, se propone un flujo del proceso contable como se muestra en la figura 3. Donde se establecen cinco momentos, que son:

1. Identificación

2. Reconocimiento

3. Medición

4. Presentación

5. Revelación

Cada uno de los momentos del proceso contable, esta normado por una NIIF o NIC, según el caso. Esta normativa establecerá el método de valuación de cada cuenta, así como las políticas contables, controles y registros, para su posterior presentación en los estados financieros o revelaciones en sus notas.

\section{Figura 3}

\section{Proceso Contable normativa NIIF}

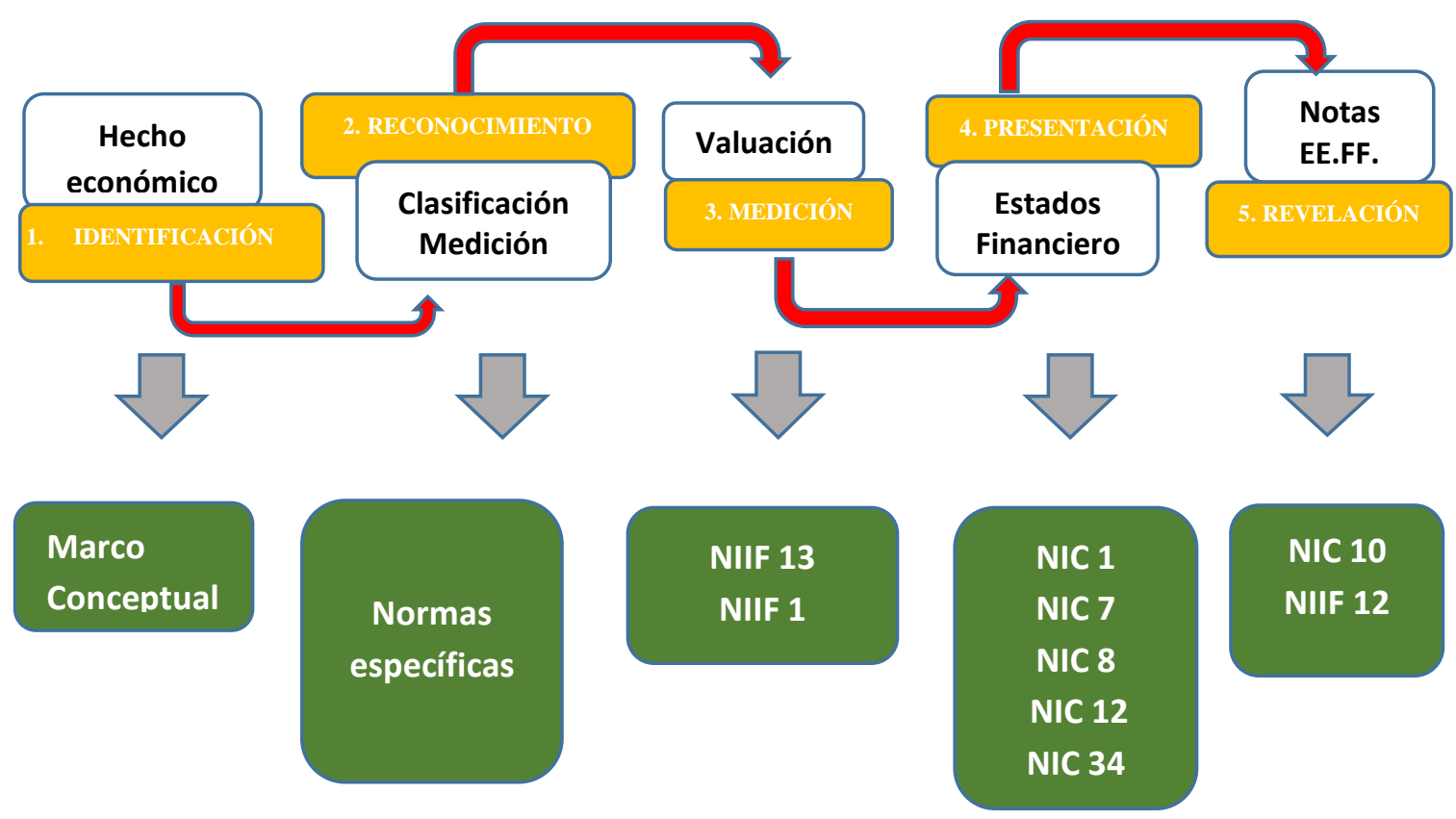

Nota: Las normas generales se encuentran presente en todo momento del proceso contable.

Fuente: Elaboración propia.

Al ser las normas generales las que determinan las políticas principales de la entidad económica, son las normas específicas las que controlan de forma individual las cuentas de los balances. La NIC 2 de inventarios por ejemplo establecerá el control para esta cuenta, estableciendo desde el método de valoración, control, registro, ajuste, entre otros procesos propios de la cuenta. 
En la figura 4 presentamos un ejemplo del proceso contable de una norma específica para el control de la cuenta de activos biológicos.

\section{Figura 4}

Proceso contable de activos bilógicos normativa NIIF.

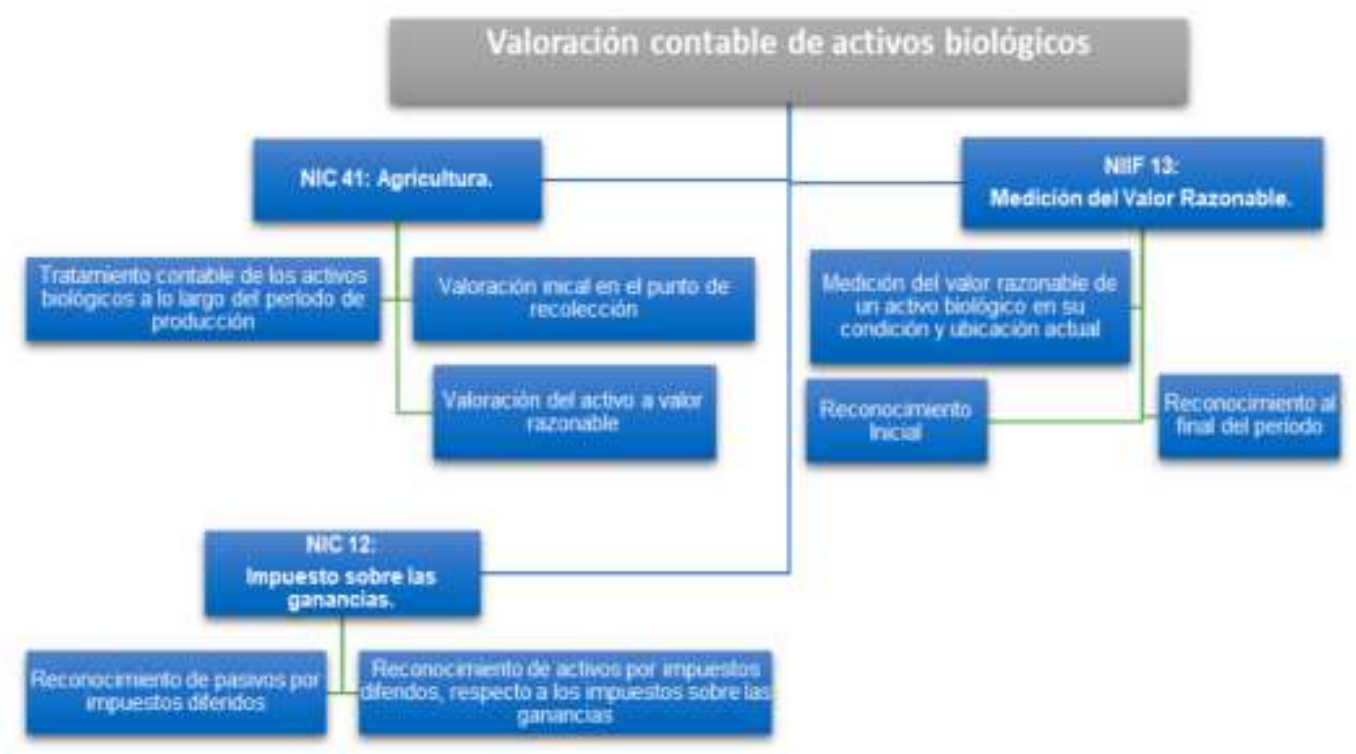

Nota: Las diversas partidas contables, generan procesos contables específicos según la normativa NIIF.

Fuente: Reyes et al, 2019.

Como se observa en la figura 4, la norma específica va acompañada en su proceso contable de las normas generales, para que exista uniformidad en la aplicación de las políticas contables y coherencia en la presentación de los estados financieros.

\section{Conclusiones}

- Al hablar de un proceso contable, podemos afirmar con lo expuesto que no se trata de un simple manual de políticas contables. El proceso contable con normativa NIIF, establece el correcto registro, valoración y presentación de cada una de las cuentas de los estados financieros, logrando así que la información plasmada sea valiosa para la toma de decisiones y fiable para sus usuarios.

- El proceso contable en NIIF no es rígido, es evolutivo y no puede usarse un mismo proceso contable para varias empresas, aunque pertenecieran a la misma rama económica. Cada entidad es única y debe ser analizada para seleccionar las mejores políticas contables que se adapten a la realidad.

- La contabilidad de una empresa a otra que se dedique a la misma actividad económica, puede variar significativamente, dependiendo del método de valoración y registro adoptado en el proceso contable. 


\section{Referencias Bibliográficas}

Albero Carballosa, C., Prado Chaviano, E., Celestrín Penabades, C., Jiménez Valero, B., \& Velastegui López, L. E. (2021). Acciones para la formación inicial de la competencia profesional específica registrar hechos económicos. Visionario Digital, 5(2), 216-224. https://doi.org/10.33262/visionariodigital.v5i2.1720

Barreto, J. y Teodomiro E. (2018). Análisis de los procesos operacionales y contables desarrollados en la empresa. Lima: Soluciones Empresariales EIRL Trujillo.

Fierro, A. y Fierro, F. (2016). Contabilidad de activos con enfoque NIIF para las PYMES. Bogotá: Ecoe ediciones.

Macedonio, Alanís y García, Pablo (2020). Tecnología de la información y la práctica contable. ISBN-13: 979-8-6212-4443-9

Amat, Oriol y Puig, Xavier. (2018). Master en Finanzas: Claves, fundamentos, estrategias y operativas de las finanzas empresariales. Profit Editorial I.

Normas Internacionales de información Financiera NIIF (2021), https://www.ifrs.org/

Normas Internacionales de información Financiera NIIF (2021), https://www.ifrs.org/use-around-the-world/why-global-accounting-standards/

Palella Santa y Martins Feliberto. (2012). Metodología de la investigación cuantitativa (1ra reimpresión). Caracas: Fedupel ISBN: 980-273-445-4.

Reyes Arana, M. Y., Narváez Zurita, C. I., Andrade Amoroso, R. P., \& Erazo Álvarez, J. C. (2019). Valoración contable de activos biológicos bajo NIIF en la empresa camaronera Biotónico S.A. Visionario Digital,3(2.1.), 476-496. https://doi.org/10.33262/visionariodigital.v3i2.1..585

Valdez Fajardo, W. P., Narváez Zurita, C. I., Ormaza Andrade, J. E., \& Erazo Álvarez, J. C. (2019). Procedimientos contables y su incidencia en la sistematización de la información de la Cooperativa de Ahorro y Crédito Atlántida de la ciudad de Cañar. Visionario Digital, 3(2.1.),

497-526. https://doi.org/10.33262/visionariodigital.v3i2.1..586

Vazquez, Nitzia y Díaz, Manuel. (2013). Normas Internacionales de Informacion Financiera (NIIF-IFRS). Profit Editorial.

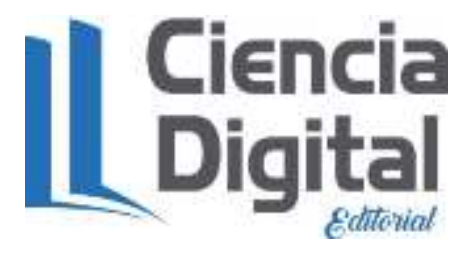




\section{PARA CITAR EL ARTÍCULO INDEXADO.}

Toledo Castillo, N. del R., Peñafiel Moncayo, I. R., \& Carrasco Ruano, Y. T. (2021). Las NIC - NIIF dentro del proceso contable. AlfaPublicaciones, 3(3.1), 57-67. https://doi.org/10.33262/ap.v3i3.1.77

\section{Ciencia \\ Digital \\ Editovial}

El artículo que se publica es de exclusiva responsabilidad de los autores y no necesariamente reflejan el pensamiento de la Revista Alfa Publicaciones.

El artículo queda en propiedad de la revista y, por tanto, su publicación parcial y/o total en otro medio tiene que ser autorizado por el director de la Revista Alfa Publicaciones.
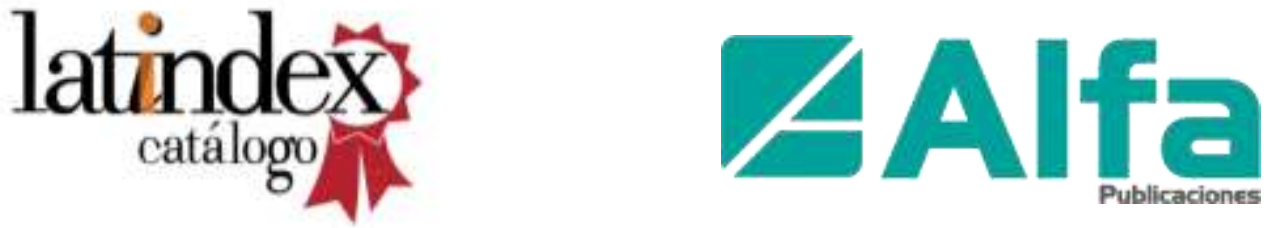